\title{
LIBERAÇÃO CONTROLADA DO FÁRMACO MEBENDAZOL A PARTIR DE UMA MATRIZ POLIMÉRICA NATURAL
}

\author{
Priscila Pereira Silva-Caldeira ${ }^{*}$ \\ Andressa Castro Terto Vilas Boas* \\ Bárbara Paiva Machado** \\ Diogo Émerson Leite de Carvalho*** \\ Kláudia Maria Machado Neves Silva ${ }^{* *+*}$
}

\begin{abstract}
RESUMO: Sistemas de liberação controlada de fármacos têm por objetivos principais minimizar a necessidade de doses tóxicas e propiciar melhor controle da liberação do princípio ativo ao longo do tempo quando comparado à forma convencional de administração. Neste trabalho foi preparado um sistema de liberação controlada do fármaco mebendazol (MDZ), que apresenta baixa absorção gastrointestinal pela sua baixa solubilidade em água, a partir de uma matriz polimérica biodegradável formada por alginato de sódio. 0 teste de intumescimento e a avaliação temporal da liberação do ativo in vitro foram realizados em meio que simula as condições do corpo humano. A avaliação da resistência ao meio fisiológico pela matriz polimérica com MDZ incorporado mostrou que a mesma permaneceu por mais de $72 \mathrm{~h}$ no meio fisiológico, enquanto que a avaliação do comportamento de dissolução e liberação do MDZ durante $8 \mathrm{~h}$ mostrou-se satisfatória com a liberação do ativo de forma lenta de gradual, 0 que demonstra o potencial dessa matriz para ser usado como um dispositivo oral de liberação controlada desse fármaco.
\end{abstract}

PALAVRAS-CHAVE: Liberação controlada de fármacos; Mebendazol; Alginato.

\section{CONTROLLED RELEASE OF THE MEBENDAZOL DRUG FROM A NATURAL POLYMERIC MATRIX}

\begin{abstract}
The main objectives of controlled drug release system are to minimize the need for toxic doses and to provide better control of the active principle release over time when compared to the conventional form of drug administration. In this work, a controlled release system for the drug mebendazole (MDZ) was prepared using a biodegradable polymeric matrix formed by sodium alginate. This drug has low gastrointestinal absorption due to its low solubility in water. The swelling test and time evaluation of drug release in vitro were performed in a medium that simulates the conditions of the human body. The evaluation of MDZ-doped polymer matrix resistance in the medium showed that it remained for more than $72 \mathrm{~h}$ in the physiological environment, while the evaluation of the dissolution and release behavior of the MDZ for 8h showed a satisfactory result with a slowly and gradual release of the drug, which indicates the matrix's potential for being used as a oral device for controlled drug delivery.
\end{abstract}

KEYWORDS: Drug controlled release; Mebendazole; Alginate.

\footnotetext{
* Docente permanente do Programa de Pós-Graduação Multicêntrico em Química de Minas Gerais no CEFET-MG (PPGMQ-MG/CEFET-MG. Docente permanente do Mestrado interdisciplinar em Tecnologia de Produtos e Processos do CEFET-MG, Brasil.

** Graduando em Química Tecnológica Centro Federal de Educação Tecnológica de Minas Gerais, CEFET/MG, Brasil.

${ }^{* * *}$ Graduando em Química Tecnológica Centro Federal de Educação Tecnológica de Minas Gerais, CEFET/MG, Brasil.

${ }^{* * * *}$ Mestrando em Química na Universidade Federal de Ouro Preto (UFOP), Brasil.

${ }^{* * * * *}$ Doutoranda em Engenharia de Produção na Universidade Federal de Minas Gerais (UFMG), Brasil.
} 


\section{INTRODUÇÃo}

$\mathrm{Na}$ administração convencional de fármacos, a concentração da droga na corrente sanguínea aumenta até atingir 0 nível máximo de dissolução e então decresce, sendo necessária a administração de nova dose para manter os níveis de concentração terapêuticos. Por outro lado, sistemas de liberação controlada de fármacos têm ampla vantagem sobre as formas de dosagem convencionais, incluindo maior eficácia dado ao maior controle da liberação do princípio ativo ao longo do tempo e toxicidade reduzida, uma vez que a administração de doses elevadas não é necessária (KAMALY et al., 2016). Somado a isso, a liberação controlada de um agente terapêutico pode, além de otimizar a resposta farmacológica e evitar fenômenos de sub ou superdosagem, prevenir reações indesejáveis, como a irritação gástrica. Tais sistemas geralmente usam matrizes poliméricas naturais ou sintéticas como transportadores dos princípios ativos de interesse. Embora 0 uso de polímeros sintéticos seja mais comum em dispositivos de liberação de fármacos, os naturais têm a vantagem de apresentar alta estabilidade química, biocompatibilidade, biodegradabilidade e capacidade de formar hidrogéis (KAVIANINIA et al., 2016). De fato, são encontrados muitos estudos na literatura que avaliam matrizes poliméricas como alternativa para modificação do perfil de liberação de diversos fármacos. Dentre os polímeros naturais conhecidos, 0 alginato de sódio (Alg) é um dos biopolímeros mais comumente utilizados, dado que possui propriedades físico-químicas adequadas para 0 desenvolvimento de sistemas de liberação controlada por via oral (AGARWAL et al., 2015). Além disso, esse biopolímero pode formar hidrogéis que apresentam intumescimento dependente do $\mathrm{pH}, \mathrm{o}$ que se configura como um fator chave no preparo de dispositivos para administração oral de fármaco ao longo do trato gastrointestinal (ABD EL-GHAFFAR et al., 2012).

0 mebendazol, (MDZ), N-metil-5-(6)benzoil-2-benzimidazol-carbamato (Figura 1), é um fármaco de amplo espectro anti-helmíntico usado em todo 0 mundo para tratamento de diferentes parasitoses em humanos e em animais (SAIDMANA et al., 2019; ZHANG et al., 2019). Estudos recentes também têm demonstrado a potencialidade de aplicar o MDZ para tratamento de cânceres. Alguns estudos indicam que 0 mebendazol possui efeito antitumoral sem apresentar toxicidade significativa para células normais (PANTZIARKA et al., 2014). Esse princípio ativo é usualmente comercializado como comprimidos ou em suspensões para administração oral. A biodisponibilidade de medicamentos administrados por via oral é determinada por vários fatores, sendo 0 mais importante a solubilidade em água e permeabilidade intestinal. 0 mebendazol, por sua vez, por ser praticamente insolúvel em água, apresenta má absorção no trato intestinal (ZHANG et al., 2019).

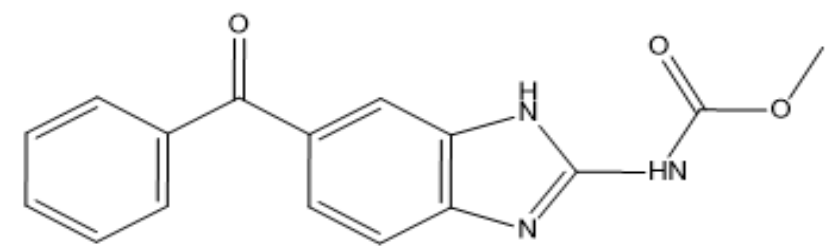

Figura 1. Estrutura química do Mebendazol

0 mebendazol apresenta-se como uma moléculabastanteresistente,sendopredominantemente eliminado na forma inalterada na urina e bile (GHAFIL et al., 2017). 0 MDZ apresenta como vantagem o fato de possuir baixa toxicidade, embora os pacientes possam sofrer de sintomas transitórios, como dor abdominal e diarreia. No caso da administração a longo prazo, a evidência é que, em geral, 0 tratamento é bem tolerado (PANTZIARKA et al., 2014). 0 metabolismo do MDZ garante que apenas cerca de $20 \%$ da dose oral atinja a circulação sistêmica e a concentração máxima é atingida no plasma entre 2 e 4 horas após administração (PANTZIARKA et al., 2014). A baixa insolubilidade em fluidos biológicos do mebendazol tem sido reconhecida e várias estratégias têm sido empregadas a fim de melhorar a biodisponibilidade desse fármaco. Entre elas pode-se citar: formulações alternativas com óleos vegetais, alteração da estrutura cristalina de MDZ e encapsulação em cilodextrinas (SAIDMANA et al., 2019). Uma opção que pode ser empregada também é 
a dispersão do fármaco em um polímero biodegradável (GHAFIL et al., 2017; KRISHNAIAH et al., 2001) que permite que o fármaco seja liberado gradualmente impedindo a saturação do sistema e proporcionando a redução do número de doses.

0 alginato é um polissacarídeo extraído de algas marinhas constituído pelos ácidos -D-manurúrico (M) e -L-gulurônico (G), no qual a proporção entre esses monômeros (M/G) difere dependendo da fonte natural (HAN; WANG, 2017; TONG et al., 2008). Este biopolímero é amplamente utilizado para 0 desenvolvimento de sistemas controlados de liberação de fármacos, engenharia de tecidos, encapsulamento de células, além de outras aplicações, pela sua baixa imunogenicidade, natureza não tóxica e capacidade de formar estruturas tridimensionais reticuladas (REN et al., 2016). Na presença de cátions divalentes como o cálcio, resíduos gulurônicos adjacentes podem interagir com esses cátions formando uma estrutura denominada "modelo caixa de ovos", conforme representado na Figura 2. de hidrogênio entre as cadeias poliméricas predomina sobre as interações polímero-água. Por outro lado, em meio neutro, a maior parte dos grupamentos ácido carboxílico é ionizada $\left(-\mathrm{COO}^{-}\right)$, uma vez que 0 pKa desses grupos no polissacarídeo é quase 3,2 (HOOGENDAM et al., 1998). Assim, as interações de ligação de hidrogênio entre as cadeias poliméricas são enfraquecidas e a repulsão eletrostática dos grupos C00- resultam em maior taxa de intumescimento e consequentemente liberação de fármaco (SILVA et al., 2019).

Objetivando ampliar a absorção do MDZ no intestino, esse trabalho descreve o preparo e o estudo da liberação desse princípio ativo a partir de uma matriz polimérica constituída por alginato de sódio. Foram realizados estudos de intumescimento e avaliação do comportamento de dissolução e liberação do MDZ a partir da matriz polimérica durante $8 \mathrm{~h}$ a fim de avaliar a potencialidade do sistema preparado.

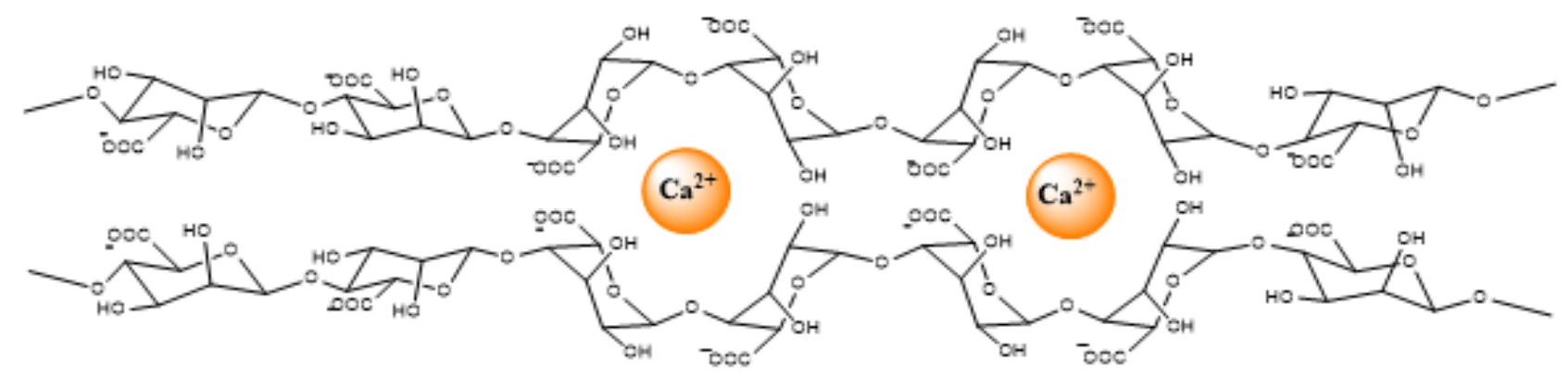

Figura 2. Representação de estruturas "modelo caixa de ovos" resultantes da interação de alginato com cátions divalentes de cálcio

Numa via oral de administração, a forma de dosagem é dirigida para o estômago, onde reside por um período definido, depois é conduzida para 0 intestino delgado e finalmente para o cólon (BAJPAl; SAXENA; SHARMA, 2006). Uma vez que 0 alginato é resistente em meio ácido e decompõe-se lentamente em meio neutro ou básico, esse biopolímero apresenta propriedades apropriadas para ser usado como sistema de liberação de fármaco no intestino. Em meio ácido, a maioria dos grupos ácido carboxílicos do alginato existe na forma protonada $(\mathrm{COOH})$, na qual a ligação

\section{METODOLOGIA}

\subsection{MATERIAIS}

Alginato de sódio (SA, viscosidade: 300 - 400 mPa.s, $180 \mu \mathrm{m})$, adquirido da empresa Êxodos Científica $®$ (Brasil) e o cloreto de cálcio anidro adquirido da VETEC $®$ (Brasil), foram utilizados sem prévia purificação. 0 mebendazol foi extraído e purificado a partir de comprimidos Belmirax $®$ (Belfar - Brasil). 


\subsection{INSTRUMENTAÇÃO}

0 espectro de absorção na região do infravermelho $\left(4000400 \mathrm{~cm}^{1}\right)$ do mebendazol foi obtido em um espectrômetro FTIR Perkin-Elmer 283B. A análise elementar referente aos teores de carbono, hidrogênio e nitrogênio do mebendazol obtido por extração foi obtida com auxílio de um equipamento Perkin Elmer modelo 2400. A temperatura de fusão do MDZ extraído foi verificada em um aparelho digital da Microquímica, MQAPF-302. A quantidade de mebendazol liberado da matriz polimérica ao longo do tempo foi determinada por absorção na região ultravioleta-visível com auxílio de um espectrofotômetro Varian Cary 50. A análise térmica das esferas de alginato dopadas com MDZ foi realizada em um equipamento Shimadzu DTG-60H a uma taxa de aquecimento de $10^{\circ} \mathrm{C} \mathrm{min}{ }^{1} \mathrm{em}$ uma atmosfera de ar sintético (50 $\left.\mathrm{mL} \mathrm{min}^{1}\right)$.

\subsection{PROCEDIMENTOS}

\subsubsection{Extração do Mebendazol}

0 mebendazol foi obtido a partir de comprimidos da marca Belmirax ${ }^{\circledR}$ (100 mg de MDZ por comprimido). Para extração do princípio ativo, dez comprimidos foram macerados e adicionados a $40 \mathrm{~mL}$ de ácido fórmico. 0 sistema foi mantido em agitação e aquecimento a $80^{\circ} \mathrm{C}$ por 30 minutos. Após atingir temperatura ambiente, 0 sistema foi filtrado e 0 sólido desprezado. À solução-mãe foram adicionados $50 \mathrm{~mL}$ de água destilada e 0 sistema foi mantido resfriado, aproximadamente $8^{\circ} \mathrm{C}$, por 24 horas. 0 sólido formado foi filtrado, lavado abundantemente com água e encaminhado para análise de espectroscopia na região do infravermelho, análise elementar e determinação da temperatura de ponto de fusão que confirmaram a obtenção do MDZ puro.

\subsubsection{Preparo das Esferas de Alginato Dopadas com MDZ}

Em um béquer foram adicionados $5 \mathrm{~g}$ de alginato de sódio em $100 \mathrm{~mL}$ de água e 0 sistema permaneceu em agitação magnética até completa dissolução do alginato. Posteriormente foi adicionado MDZ equivalente a $20 \% \mathrm{~m} / \mathrm{m}$ e 0 sistema foi mantido em agitação por mais 30 minutos para completa incorporação do princípio ativo na matriz polimérica. Em seguida, esferas poliméricas foram obtidas a partir da adição de gotas da solução polimérica resultante em uma solução $2 \%$ de cloreto de cálcio por 15 minutos. As esferas formadas foram então coletadas da solução e colocadas em estufa para completa secagem $\left(40^{\circ} \mathrm{C}\right.$ por $15 \mathrm{~h}$ ). As esferas de alginato com MDZ incorporado foram separadas para testes de intumescimento, liberação de fármaco e análise térmica.

\subsubsection{Teste de Intumescimento in vitro}

0 comportamento de inchamento das esferas de alginato incorporadas com MDZ foi estudado na temperatura fisiológica $\left(37^{\circ} \mathrm{C}\right)$ imergindo amostras secas para inchar em $2 \mathrm{~mL}$ de tampão fosfato $(\mathrm{pH} 7,4)$. Em intervalos de tempo específicos, as amostras foram removidas do meio de intumescimento, o excesso de umidade da superfície retirado com auxílio de um pedaço de papel toalha e a massa dos hidrogéis dilatados foi medida em uma balança eletrônica. As medições foram feitas em triplicada e os dados médios foram usados para cálculos. A massa inchada foi obtida de acordo com a relação empírica, Eq. (1), em que $m_{f}$ é a massa final e m é a massa da amostra seca.

$$
\% \text { inchamento }=\frac{m_{f}-m_{0}}{m_{0}} \times 100
$$




\subsubsection{Teste de Liberação do Princípio Ativo in vitro}

Para simular o perfil de liberação do fármaco no intestino, esferas carregadas com MDZ foram incubados em $10 \mathrm{~mL}$ de solução tampão $(\mathrm{pH}=7,4)$ a $37^{\circ} \mathrm{C}$ em sistema com agitação contínua. Nos intervalos de tempo predeterminados, $300 \mu \mathrm{L}$ do sobrenadante foram retirados e substituídos por uma solução tampão nova. A quantidade de MDZ foi determinada a $290 \mathrm{~nm}$ utilizando um espectrofotômetro de absorção na região do UV-vis. A porcentagem da quantidade acumulada de MDZ liberada foi avaliada a partir da curva de calibração padrão. A curva de calibração em pH 7,4 foi obtida pela variação das concentrações de MDZ de 0,7 a 12,0 mg L ${ }^{1}$, cuja equação de regressão linear é dada por $y=6,43 x+0,133\left(R^{2}=0,96\right)$. Não foi observada presença de produtos de degradação do MDZ. Os experimentos de liberação in vitro foram realizados em triplicata.

\subsubsection{Preparo da Solução Tampão PBS em pH=7,4}

Para simular as condições fisiológicas foi preparada solução tampão de $\mathrm{pH}=7,4$. A solução tampão foi preparada nas seguintes proporções molares: 1,37M de cloreto de sódio, 26,8mM de cloreto de potássio, 0,1M de fosfato de sódio mono-hidratado e 17,6mM de fosfato de potássio em cerca de $800 \mathrm{~mL}$ de água destilada. $0 \mathrm{pH}$ foi ajustado com adição de solução de hidróxido de sódio $5 \mathrm{M}$ até que se atingisse 0 pH 7,4. 0 volume foi, então, completado com água destilada para $1 \mathrm{~L}$ em um balão volumétrico.

\section{RESULTADOS E DISCUSSÃO}

0 mebendazol pode apresentar-se em três formas polimórficas distintas nomeadas como $A, B$ e C (BUNACIU; FLESCHIN; ABOUL-ENEIN, 2002). A análise de espectroscopia de absorção na região do infravermelho obtida para 0 mebendazol extraído (Figura 3) indicou tratar-se da forma polimórfica $B$ (BUNACIU; FLESCHIN; ABOUL-ENEIN, 2002). Ademais, a temperatura de fusão do MDZ extraído foi determinada no intervalo de $291294^{\circ} \mathrm{C}$, enquanto o valor descrito na literatura é $288,5^{\circ} \mathrm{C}$ (DRUG BANK, 2018). A proximidade entre os valores temperatura de fusão experimental e teórico, bem como o espectro de infravermelho do MDZ extraído indicaram que foi possível extrair o MDZ a partir dos comprimidos comerciais. 0 MDZ foi então incorporado em esferas de alginato a fim de preparar um dispositivo para liberação controlada desse fármaco.

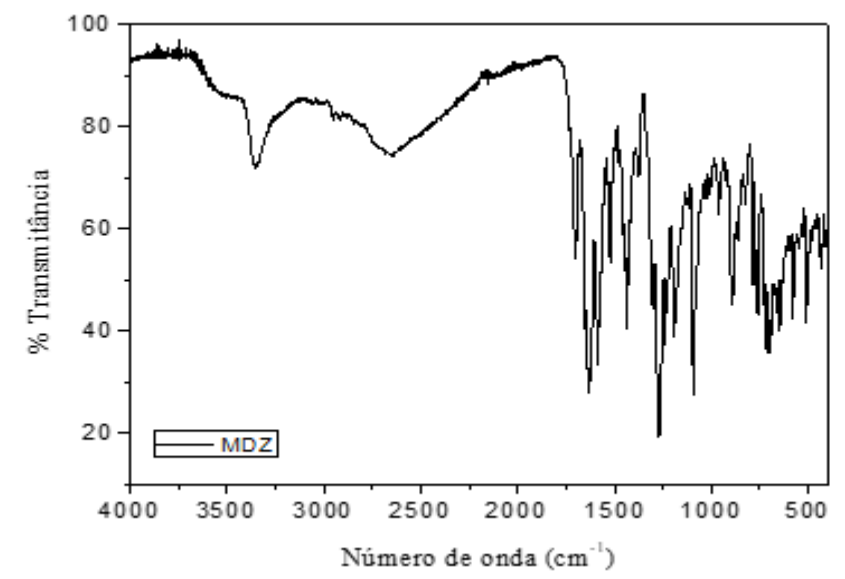

Figura 3. Espectro de absorção na região do infravermelho do mebendazol extraído

0 teste de intumescimento é importante uma vez que a biodegradação de matrizes poliméricas influencia diretamente na cinética de liberação do fármaco. Dessa forma, a integridade da forma farmacêutica é um dos fatores determinantes a ser considerado para se evitar uma degradação prematura do transportador. A Figura 4 apresenta 0 resultado da avaliação da resistência da matriz polimérica ao tampão fisiológico $(\mathrm{pH}=7,4)$. Nas primeiras 06 horas a matriz inchou cerca de $64 \%$ e essa proporção de absorção de água foi mantida praticamente constante durante 72 horas, mostrando que a matriz alcançou um ponto de equilíbrio de absorção de água e permaneceu estável por um longo período. Dessa forma pode-se inferir que, por causa do processo lento de difusão do líquido e de degradação do polímero no meio, a liberação total deste medicamento ocorrerá em um tempo mínimo de três dias. 


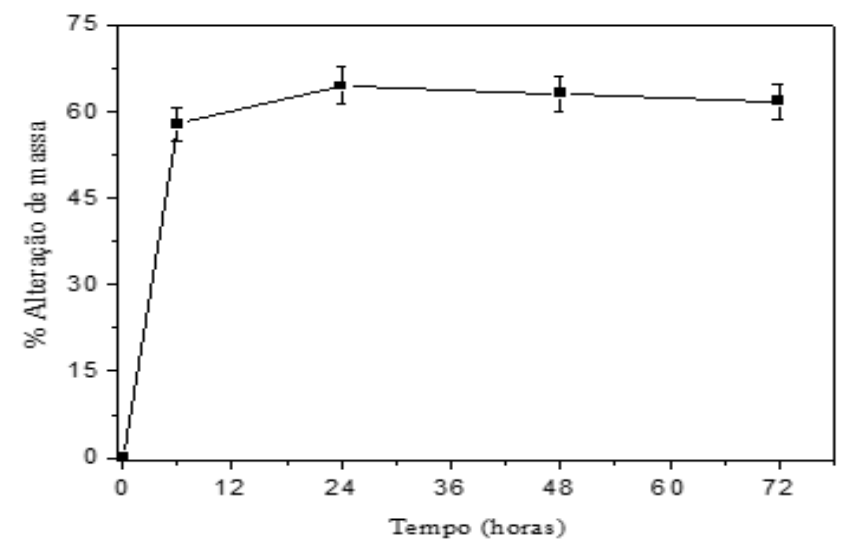

Figura 4. Teste de intumescimento em $\mathrm{pH}=7,4 \mathrm{~A} 37^{\circ} \mathrm{C}$.

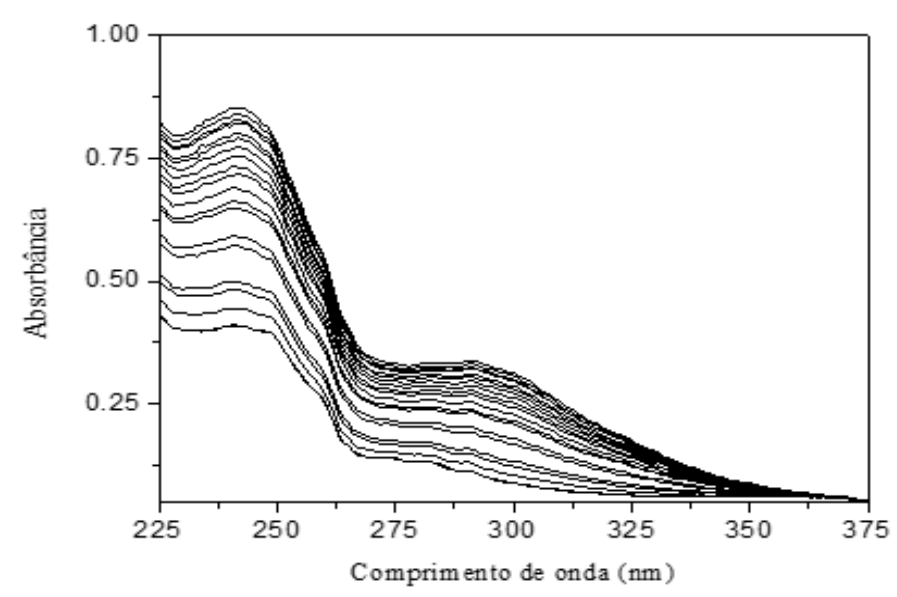

Figura 5. Espectros de absorção na região do UV-Vis do mebendazol em $\mathrm{pH}=7,4$. Concentração variando de 0,7 a $12,0 \mathrm{mg} \mathrm{L}^{1}$

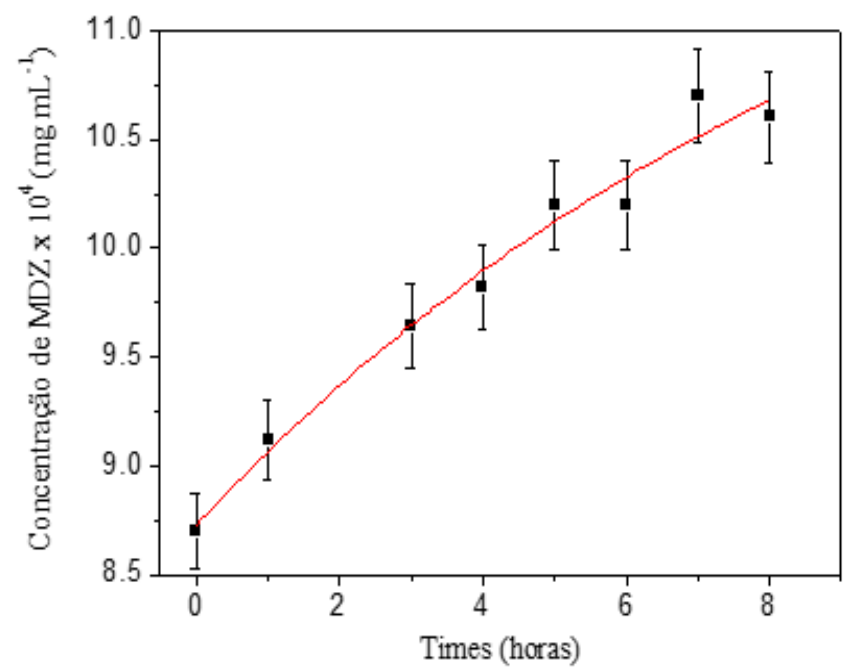

Figura 6. Curva de liberação controlada do MDZ em função do tempo
0 mebendazol possui duas absorções na região do ultravioleta-visível uma a $240 \mathrm{~cm}^{1}$ e outra a $290 \mathrm{~cm}^{1}$ (Figura 5). A curva de calibração foi construída utilizando os valores de absorção obtidos a $290 \mathrm{~cm}$. A avaliação temporal da liberação do princípio ativo in vitro foi conduzida avaliando-se o comportamento de dissolução e liberação do mebendazol por um período de $8 \mathrm{~h}$ em um meio que simula as condições do corpo humano ( $\mathrm{pH}=7,4)$, como demonstrado na Figura 6 . 0s comprimidos convencionais de mebendazol liberam 0 princípio ativo ao longo de todo o trato gastrointestinal e, portanto, causam efeitos colaterais indesejáveis (KRISHNAIAH et al., 2001). 0 direcionamento do mebendazol para ação local, apenas no cólon, pode ser benéfico para evitar efeitos indesejados. Dessa forma, uma matriz composta por alginato que não se degrada em meio ácido (HOOGENDAM et al., 1998), como 0 estômago, e sofre erosão lenta e gradual em meio neutro, como o cólon, é interessante para liberação controlada do MDZ. É possível observar que a liberação do ativo ocorre de forma gradual no $\mathrm{pH}$ correspondente ao do cólon, pH=7,4, (Figura 6). Essa liberação lenta e gradual do fármaco é interessante, pois além de impedir que ocorra a saturação do fármaco no sistema, o MDZ mesmo em pequenas doses é potente para ação farmacológica almejada (KRISHNAIAH et al., 2001).

A liberação contínua de fármacos a partir de matrizes poliméricas pode ocorrer por difusão dos fármacos da matriz ou pela erosão do polímero (pela degradação) ou pela combinação de processos de erosão e difusão (FU; KAO, 2011). Como a matriz polimérica apresenta certa resistência à degradação em tampão com pH fisiológico $(7,4)$ durante as primeiras $8 \mathrm{~h}$, conforme observado pelo teste de intumescimento, dado à ausência de picos de liberação do fármaco e à baixa solubilidade do MDZ, propõe-se nesse estudo uma combinação dos processos de difusão e erosão como principais forças para a liberação do mebendazol (FU; KAO, 2011). 


\section{CONCLUSÃO}

Neste estudo, demonstramos que as esferas de alginato incorporadas com mebendazol é um candidato potencial para a liberação oral desse agente terapêutico, uma vez que este sistema permite uma liberação controlada do fármaco ao longo do tempo. Além disso, o teste de intumescimento corroborou com esse resultado mostrando que esse sistema é resistente ao meio fisiológico por pelo menos $72 \mathrm{~h}$ e, portanto, adequado para utilização como sistema de liberação controlada uma vez que não se degrada completamente nas primeiras horas.

\section{AGRADECIMENTOS}

Nós agradecemos ao Centro Federal de Educação Tecnológica de Minas Gerais (CEFET-MG), Conselho Nacional de Pesquisa (CNPq), à Fundação de Amparo a Pesquisa do Estado de Minas Gerais (FAPEMIG), e à Coordenação de Aperfeiçoamento de Pessoal de Nível Superior - Brasil (CAPES) pelo suporte financeiro.

\section{REFERÊNCIAS}

ABD EL-GHAFFAR, M. A.; HASHEMA, M. S.; ELAWADYB, M. K.; RABIEC, A. M. PH-sensitive sodium alginate hydrogels for riboflavin controlled release. Carbohydrate Polymers, v. 89, n. 2, p. 667-675, 2012.

AGARWAL, T. et al. Calcium alginate-carboxymethyl cellulose beads for colon-targeted drug delivery. International Journal of Biological Macromolecules, v. 75, p. 409-417, 2015.

\section{BAJPAI, S. K.; SAXENA, S. K.; SHARMA, S. Swelling} behavior of barium ions-crosslinked bipolymeric sodium alginate-carboxymethyl guar gum blend beads. Reactive and Functional Polymers, v. 66, $\mathrm{n}$. 6, p. 659-666, 2006.

BUNACIU, A. A.; FLESCHIN, S.; ABOUL-ENEIN, H. $Y$. Analysis improvement of drugs using modern methods. Romanian Biotechnological Letters, v. 7, n. 2, p. 647654, 2002.

MEBENDAZOLE. In: DrugBank Database [Internet]. Disponível em: https://www.drugbank.ca/drugs/ DB00643. Acesso em: 23 abr. 2019.

FU, Y.; KA0, W. J. Drug release kinetics and transport mechanisms of non- degradable and degradable polymeric delivery systems. Expert Opinion Drug Delivery Journal, v. 7, n. 4, p. 429-444, 2011.

GHAFIL, F.; ANUTA, V.; SARBU, L.; TODERESCU, C. D.; MIRCIOIU, I. Increasing the bioavalilability of mebendazole influence of croscarmellose on dissolution rate, extent and mechanism in simulated gastric medium. Vasile Goldis University Press, v. 27, n. 1, p. 69-78, 2017.

HAN, Y.; WANG, L. Sodium alginate / carboxymethyl cellulose films containing pyrogallic acid: physical and antibacterial properties. Journal of the Science of Food and Agriculture, v. 97, p. 1295-1301, 2017.

HOOGENDAM, C. W. et al. Persistence length of carboxymethyl cellulose as evaluated from size exclusion chromatography and potentiometric titrations. Macromolecules, v. 31, n. 18, p. 62976309, 1998.

KAMALY, N.; YAMEEN, B.; WU, J.; FAROKHZAD, 0. C. Degradable controlled-release polymers and polymeric nanoparticles: Mechanisms of controlling drug release. Chemical Reviews, v. 116, n. 4, p. 2602-2663, 2016.

KAVIANINIA, I. et al. Design and evaluation of a novel chitosan-based system for colon-specific 
drug delivery. International Journal of Biological

Macromolecules, v. 85, p. 539-546, 2016.

KRISHNAIAH, Y. S. R.; VEER RAJU, P.; DINESH KUMAR, B.; BHASKAR, P.; SATYANARAYANAA, V. Development of colon targeted drug delivery systems for mebendazol. Journal of Controlled Release, v. 77, p. 87-95, 2001.

PANTZIARKA, P.; BOUCHE, G.; MEHEUS, L.;

SUKHATME, V.; SUKHATME, V. P. Repurposing Drugs in Oncology (ReDO) - mebendazole as an anti-cancer agent. Cancer Medical Science, v. 8, 2014.

REN, H.; GAO, Z.; WU, D.; JIANG, J.; SUN, Y. L. Congwei Efficient $\mathrm{Pb}(\mathrm{II})$ removal using sodium alginate - carboxymethyl cellulose gel beads: preparation, characterization, and adsorption mechanism. Carbohydrate Polymers, v. 137, p. 402-409, 2016.

SAIDMANA, E. et al. Inclusion complexes of -cyclodextrin and polymorphs of mebendazole physicochemical characterization. European Journal of Pharmaceutical Sciences, v. 127, p. 330-338, 2019.

SILVA, K. M. M. N. et al. Concomitant and controlled release of furazolidone and bismuth(III) incorporated in a cross-linked sodium alginate-carboxymethyl cellulose hydrogel. International Journal of Biological Macromolecules, v. 126, p. 359-366, 2019.

TONG, Q.; XIAO, Q.; LIM, L. T. Preparation and properties of pullulan-alginatecarboxymethylcellulose blend films. Food Research International, v. 41, n. 10, p. 1007-1014, 2008.

ZHANG, L. et al. Mebendazole potentiates radiation therapy in triple-negative breast. Cancer, v. 103, n. 1, p. 195207, 2019.
Recebido em: 01/05/2019

Aceito em: 05/06/2019 